\title{
Anorexia nervosa and juvenile lupus erythematosus in a 16-year-old female patient - common disease origin or random coincidence?
}

\author{
LIDIA HYLA-KLEKOT ${ }^{l}$, AGNIESZKA WOLNY ${ }^{1}$, MAEGORZATA JANAS-KOZIK ${ }^{2,3}$, \\ TOMASZ KOSZUTSKI
}

${ }^{1}$ Department of Pediatric Surgery and Urology, Medical University of Silesia, Katowice, Poland

${ }^{2}$ John Paul II's Pediatric Centre in Sosnowiec, Sosnowiec, Poland

${ }^{3}$ Clinical Ward of Developmental Age Psychiatry and Psychotherapy, Department of Psychiatry and Psychotherapy, Medical University of Silesia, Katowice, Poland

\begin{abstract}
Adolescence is a period in which eating disorders and juvenile systemic lupus erythematosus are typically diagnosed. The coexistence of both disorders prompts the search for a common aetiology.

In this paper, we present a case of a 16-year-old girl with life-threatening anorexia nervosa followed by clinical and immunological manifestations of systemic lupus erythematosus. The severity of the symptoms of anorexia nervosa resulted in significant delay in proper diagnosis of the concomitant systemic disease which had already been active. The administration of immunosuppressive treatment resulted in decreased lupus activity and resolution of the symptoms of anorexia nervosa. Being affected by one severe and chronic disease does not preclude the coexistence of another disease of different aetiology. However, such coexistence may suggest a common pathophysiology. Many authors have indicated a possible link between anorexia nervosa and many autoimmune disorders. Currently, modern genetic techniques have confirmed a significant correlation between these disorders. This issue needs further investigation and may be helpful in arriving at the final diagnosis in similar cases.
\end{abstract}

Key words: anorexia nervosa, systemic lupus erythematosus (SLE), immunosuppressive therapy.

(Cent Eur J Immunol 2021; 46 (1): 127-132)

\section{Introduction}

An increased risk of developing autoimmune diseases in patients with eating disorders has been confirmed by large cohort studies and many clinical observations. The correlation between anorexia nervosa (AN) and coeliac disease, psoriasis, Crohn disease, thyroiditis, multiple sclerosis and type 1 diabetes has recently been demonstrated [1-3]. In these cases a predominant role of immune disorders is proposed [4].

Anorexia nervosa is a psychiatric disorder characterized by an unreasonable fear of gaining weight, attempts to lose weight to the point of starvation and a severely distorted perception of one's physical appearance. The diagnostic criteria for AN are listed in ICD-10 and DSM-V [5-7]. Weight loss is the main objective measurable parameter. Persistent attempts to reduce weight result in mood and cognitive disorders $[8,9]$. The prevalence rate for $\mathrm{AN}$ is $0.9 \%$ among girls, which indicates that the disease is a serious medical condition [10].

Systemic lupus erythematosus (SLE) is a chronic autoimmune disease with various clinical symptoms and periods of exacerbation (flares) and remission. It usually occurs in females during puberty. Juvenile SLE is usually manifested by weight loss, generalized weakness, skin lesions, joint changes, leukopenia with lymphopenia, thrombocytopenia and nephropathy. The nervous system is also affected in $22-95 \%$ of patients. In lupus headache, mood disorders and even psychosis or cognitive impairment are commonly reported [11].

Although many clinical symptoms are characteristic of both SLE and AN, the reported coexistence of SLE and $\mathrm{AN}$ is rare. Our case study may be of particular interest to psychiatrists, immunologists and paediatricians.

Correspondence: Prof. Lidia Hyla-Klekot, Department of Pediatric Surgery and Urology, Medical University of Silesia, Katowice, Poland, e-mail: lidiahylaklekot@gmail.com

Submitted: 6.08.2019; Accepted: 29.10.2019 


\section{Case report}

We present a case of a 16-year-old girl with severe AN coexisting with juvenile SLE.

The patient was a full-term newborn with normal birth weight. The girl was diagnosed with intrauterine infection which was treated with antibiotic therapy in the department of neonatology. During the first year of life, problems related to weight gain were observed. At the $11^{\text {th }}$ month of age, the girl's weight was only 8,200 $\mathrm{g}\left(<3^{\text {rd }}\right.$ percentile $)$. However, coeliac disease was ruled out. Small bowel biopsy confirmed massive eosinophilic infiltration. The patient was diagnosed with food allergy. Due to frequent hospitalisations, infant formula was immediately introduced (as a breast milk substitute) after the birth. The patient's early childhood development was normal except for speech delay.

The first alarming symptoms of an eating disorder occurred in May 2014 when the girl restricted food intake. Two years later the patient was finally diagnosed with concomitant SLE, the symptoms of which had been previously reported. The sequence of symptoms combined with the patient's body mass index (BMI) is given in Figure 1. The subject almost completely refused to eat and drink at the end of 2014 and presented with behavioural disorders (vulgarity, impulsiveness, disinhibition), self-injurious behaviour and suicidal thoughts. In June 2015, the patient's body weight decreased from 46 to $36 \mathrm{~kg}$ ( $<3^{\text {rd }}$ percentile) while her height was $155 \mathrm{~cm}$.

The patient was admitted to the department of gastroenterology for the treatment of the eating disorder. Inflammatory changes in gastric and duodenal mucosa were confirmed by endoscopic biopsy. Due to the severe eating disorder, the patient required a high-calorie diet administered by a feeding tube. The diagnosis of AN was established by a consultant psychiatrist and the patient was admitted to the department of psychiatry for further treatment.

Physical examination revealed decreased subcutaneous tissue and muscle atrophy. The weight of the patient was $35.3 \mathrm{~kg}$ (BMI $14.5 \mathrm{~kg} / \mathrm{m}^{2}$ ). The patient did not report suicidal thoughts any more. Verbal contact was maintained and psychotic symptoms were not observed. However, during the further hospital stay, the patient reported hearing voices and seeing shadows in the mirror. Brain computed tomography (CT) was unremarkable. The patient reported soreness of joints, mainly in the morning and after longer periods of rest. Subsequently, the right ankle joint was swollen and tender whereas the skin was covered with petechiae. Raynaud's phenomenon (RP) was diagnosed. Additional laboratory tests were performed due to the onset of new symptoms. Inflammatory markers were moderately elevated $(\mathrm{C}$ reactive protein $[\mathrm{CRP}] 41 \mathrm{mg} / \mathrm{dl}$, white blood cells [WBC] $\left.11.8 \times 10^{9} / 1\right)$ and the presence of antinuclear antibodies (ANAs) was observed (i.e. anti-dsDNA and anti-nucleosome antibodies). However, the possible co-occurrence of a systemic disorder was not suspected due to non-specific symptoms of an autoimmune disorder and life-threatening and exacerbating AN.

During the second month of hospitalization, the patient presented with fever of $39.8^{\circ} \mathrm{C}$. After three days of fever, skin rash occurred and finally covered the whole body. Significant facial erythema was observed. The patient reported facial itching, sore throat, nausea, and cervical and abdominal pain. Laboratory tests revealed additional

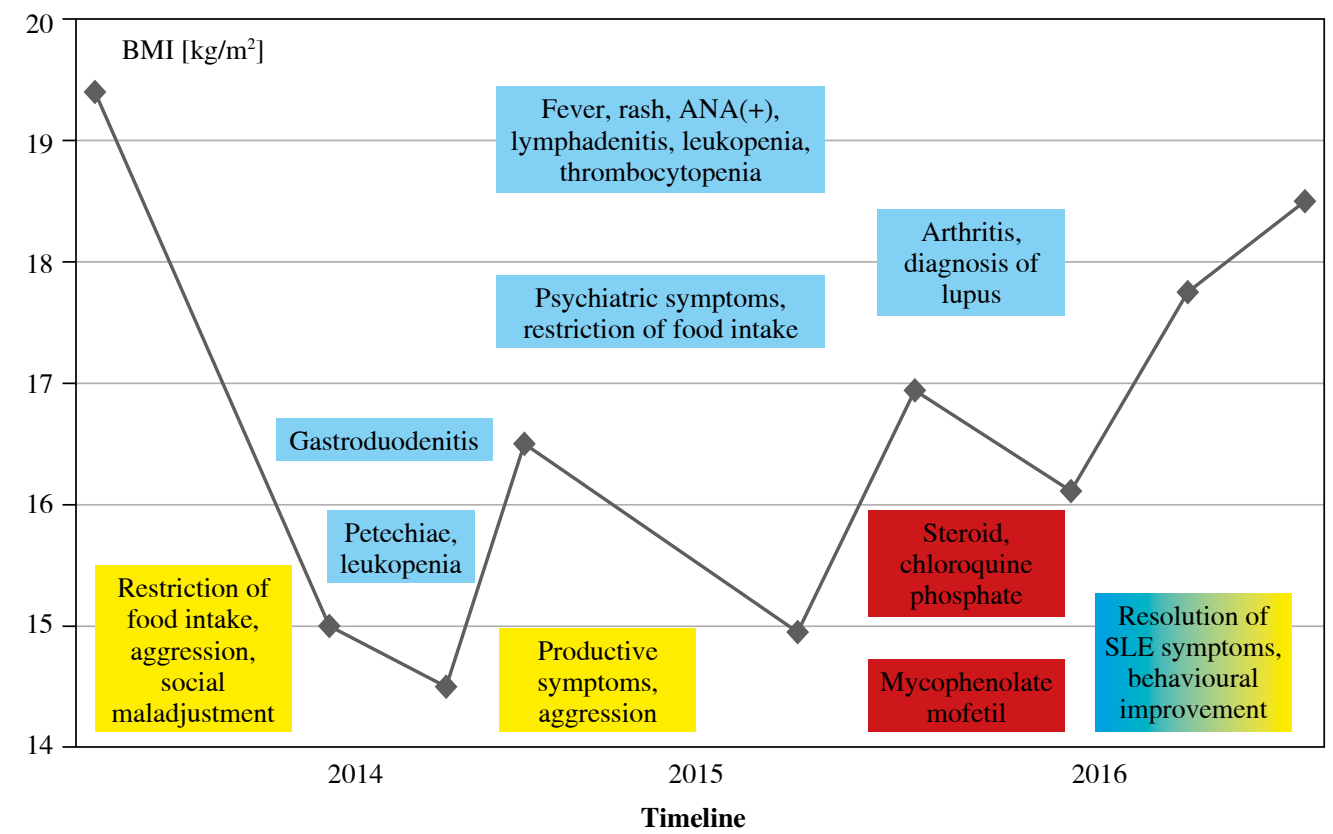

Fig. 1. Sequence of symptoms of systemic lupus erythematosus (SLE) and anorexia nervosa (AN) in association with body mass index (BMI) 
increases in inflammatory markers (CRP $59 \mathrm{mg} / \mathrm{dl}$, WBC $\left.13.1 \times 10^{9} / 1\right)$ and symptomatic treatment was introduced (antibiotic therapy). According to a consultant radiologist, the patient did not fulfil the criteria for SLE. Viral infection was diagnosed and the patient was discharged home. Slight improvement in body weight was observed during hospitalization $\left(39.4 \mathrm{~kg}\right.$; BMI $\left.16.2 \mathrm{~kg} / \mathrm{m}^{2}\right)$.

In July 2015 , the patient restricted food intake again. In November 2015, the patient was re-admitted to the department of psychiatry. On admission, her body weight was $36 \mathrm{~kg}$ (BMI $14.98 \mathrm{~kg} / \mathrm{m}^{2}$ ). Physical examination revealed no other abnormalities. Several days later the patient reported itchy urticarial lesions on the face, neck and shins. Auditory and visual hallucinations were observed. However, the body weight improved at the end of the third month of hospitalization $\left(40.7 \mathrm{~kg}\right.$; BMI $\left.16.9 \mathrm{~kg} / \mathrm{m}^{2}\right)$.

In May 2016, the patient was admitted to the department of rheumatology due to morning joint stiffness and pain. On admission, decreased subcutaneous tissue was observed.

The skin of the feet was covered with bluish lesions. Several joints (knees, left elbow and proximal interphalangeal joints of the right hand) were swollen and tender with significantly reduced mobility. Laboratory tests revealed elevated inflammatory markers (CRP $21 \mathrm{mg} / \mathrm{dl}$, WBC $\left.12.4 \times 10^{9} / 1\right)$ and an increased $\gamma$-globulin level. Other laboratory findings showed low levels of complement C3 (0.50 g/l) and C4 (0.04 g/l), the presence of ANAs $(1: 5120)$ and anti-dsDNA antibodies $(1: 1280)$. However, the titres of antibodies against cytoplasmatic components of neutrophil granulocytes (pANCA and cANCA) were negative. The ANA profile was positive for anti-SS-A, antiRo-52, anti-dsDNA, anti-nucleosome, anti-histone and anti-ribosomal $\mathrm{P}$ antibodies. Anti-cardiolipin antibodies were also present (in a low titre). A 24-hour urine collection revealed proteinuria $(0.18 \mathrm{~g} / \mathrm{d})$. The final diagnosis of SLE was made based on the whole clinical presentation.

Immediately after establishing the diagnosis, the patient was administered with oral glucocorticoid and chloroquine phosphate, which resulted in decreased joint pain and reduced skin lesions within twelve days. In June 2016, the patient was admitted to the department of nephrology due to lupus nephritis. High SLE activity was confirmed
Table 1. Selected results of the laboratory tests during immunosuppressive treatment

\begin{tabular}{lccccc}
\hline Variables & $\begin{array}{c}\text { June } \\
\mathbf{2 0 1 6}\end{array}$ & $\begin{array}{c}\text { Sep } \\
\mathbf{2 0 1 6}\end{array}$ & $\begin{array}{c}\text { Dec } \\
\mathbf{2 0 1 6}\end{array}$ & $\begin{array}{c}\text { Feb } \\
\mathbf{2 0 1 7}\end{array}$ & $\begin{array}{c}\text { May } \\
\mathbf{2 0 1 7}\end{array}$ \\
\hline Total protein $[\mathrm{g} / \mathrm{dl}]$ & 7.1 & 7.6 & 6.3 & 6.7 & 6.8 \\
\hline$\gamma$-globulins $[\mathrm{g} / \mathrm{l}]$ & 13.8 & 10.2 & 11.3 & 11.2 & 10.9 \\
\hline CRP [mg/l] & $<0.2$ & 0.25 & 0.2 & $<0.2$ & $<0.2$ \\
\hline WBC [× 10\%/l] & 8.2 & 7.1 & 4.1 & 4 & 4.27 \\
\hline \%NEUT & 84.8 & 53.2 & 44.6 & 61.1 & 56.0 \\
\hline \%LYMPH & 10.6 & 38.7 & 32.6 & 23.4 & 28.4 \\
\hline IgA [g/l] & 0.188 & 0.210 & 0.206 & & \\
\hline IgG [g/l] & 6.03 & 6.8 & 6.4 & & \\
\hline IgM [g/l] & 0.51 & 0.60 & 0.46 & & \\
\hline
\end{tabular}

by laboratory tests, which revealed decreased complement C3 $(0.70 \mathrm{~g} / \mathrm{l})$ and $\mathrm{C} 4(0.06 \mathrm{~g} / \mathrm{l})$, the presence of ANA $(1: 1280)$ and anti-dsDNA antibodies $(1: 320)$. The systemic lupus erythematosus disease activity index (SLEDAI) score was 20. Different laboratory results during immunosuppressive treatment are given in Tables 1 and 2 . Treatment with mycophenolate mofetil (MMF) was started and glucocorticoid therapy was continued. At that time no renal biopsy was performed due to the predominance of psychosomatic symptoms of AN, a severe clinical condition and low-grade proteinuria. The treatment effect of MMF was excellent. The patient's mood improved significantly after 3 months of immunosuppressive treatment. The patient's relationships with friends and family also improved considerably. In January 2017, the patient's body weight increased to $46.2 \mathrm{~kg}$ (with the same height as previously; BMI $18.9 \mathrm{~kg} / \mathrm{m}^{2}$ ). No skin or joint abnormalities were observed. Laboratory tests showed a significant reduction of SLE activity and normal urine findings.

\section{Discussion}

The severity of the symptoms of AN and progressive exacerbation of behavioural disorders and psychotic symptoms contributed to considerably delayed diagnosis

Table 2. Results of immunological tests during immunosuppressive treatment

\begin{tabular}{|c|c|c|c|c|c|}
\hline Variables & June 2016 & Sep 2016 & Dec 2016 & Feb 2017 & May 2017 \\
\hline ANA & $1: 1280$ & $1: 2560$ & $1: 1280$ & $1: 2560$ & $1: 2560$ \\
\hline Anti-dsDNA & $1: 320$ & $1: 320$ & $1: 40$ & $1: 40$ & $1: 320$ \\
\hline Anti-SS-A & +++ & +++ & +++ & +++ & +++ \\
\hline Anti-SS-B & + & + & + & 0 & + \\
\hline Anti-Ro-52 & +++ & +++ & +++ & +++ & +++ \\
\hline Anti-nucleosomes & +++ & + & +++ & ++ & ++ \\
\hline Complement C3 & 0.70 & 1.21 & 1.12 & 1.18 & 1.15 \\
\hline Complement C4 & 0.06 & 0.12 & 0.10 & 0.14 & 0.16 \\
\hline
\end{tabular}


Table 3. Clinical features common for anorexia nervosa (AN) and systemic lupus erythematosus (SLE) in a 16-year-old female patient

\begin{tabular}{l}
\hline Clinical features common for AN and SLE \\
\hline Female predominance \\
\hline Young age (10-17 years old) \\
\hline Weight loss \\
\hline Malaise, uncommon fatigue \\
\hline Mood disorders \\
\hline Cognitive disorders \\
\hline
\end{tabular}

of SLE. Despite a clear clinical manifestation and the presence of ANAs, a systemic autoimmune disease was not suspected. It was probably due to unawareness of the possible coexistence of these two diseases. Additionally, some of the patient's symptoms are simultaneously found in AN and in SLE (Table 3).

The highest prevalence of AN and SLE is observed among adolescents, and the vast majority of patients affected with these diseases are female. The prevalence of AN and SLE is 4-10 times higher among young women compared to young men [12]. The co-occurrence rate of the diseases reaches even $1 / 100$ patients [13].

The coexistence of the disorders was first reported by Babmery in 1987 (a patient with SLE developed AN during glucocorticoid therapy) [14]. A similar report was presented by Sloan 11 years later [15]. A retrospective review by Toulany et al. of 425 patients with juvenile SLE showed 7 adolescents with SLE and AN [13]. One of the patients with AN manifested SLE during treatment with prednisone. Those authors suggested that AN symptoms (including body image dissatisfaction) were likely precipitated by glucocorticoid-induced weight gain. The other 6 patients ( $1.4 \%$ of all patients) were diagnosed with AN either before or within 1-2 months of their diagnosis of SLE [13]. The short period between the manifestations of AN and SLE may indicate a common pathophysiology of both diseases.

In 2017, researchers from the King's College of London, the University of North Carolina and Stanford University identified the first genetic variant associated with AN located in chromosome 12 using the genome-wide association study (GWAS) technique. They also proved that this locus is significantly correlated with other eating disorders and psychiatric diseases such as schizophrenia [16]. The area was previously associated with type 1 diabetes and many other autoimmune disorders.

The specific aetiology of SLE is still unknown. Genetic and environmental factors as well as endocrine disorders are involved in the aetiology. The complex pathophysiology of the disease involves immune system disorders such as production of autoantibodies, the loss of immune tol- erance and disorders of apoptosis [17]. Impaired immune regulation occurs many years before the clinical onset of SLE [18]. Swedish authors reported a significantly higher risk of developing SLE in patients with AN and suggested that the coexistence of these disorders was indirectly related to the presence of brain-reactive autoantibodies. This group of antibodies is responsible for the development of SLE in patients with mental disorders (e.g. schizophrenia) and neuropsychiatric symptoms in patients with SLE $[19,20]$. Massive production of autoantibodies is found in SLE patients. However, only a small percentage of them is detected. Some of them may include antibodies against neurotransmitters, e.g. neuropeptides associated with appetite regulation or food intake. Immunological examination of patients with AN contributed to the detection of autoantibodies against proteins involved in the regulation of appetite, emotions, stress response and neuroticism [ $\alpha$-melanocyte-stimulating hormone ( $\alpha$-MSH), adrenocorticotropic hormone (ACTH), ghrelin, oxytocin]. Festissov et al. reported that a significant group of patients with eating disorders had $\alpha-\mathrm{MSH}$ and ACTH antibodies, which are involved in the control of appetite. The presence of those antibodies is considered to be a marker of immune dysfunction in AN [19, 20]. Despite the fact that the criteria for SLE do not include eating disorders, the symptoms in our patient may suggest that AN is one of the possible manifestations of neuropsychiatric SLE. The involvement of the central nervous system is poorly understood.

The possibility of the occurrence of RP in both diseases deserves attention. Although it is often found in systemic connective tissue diseases, its occurrence in AN is rarely reported [21]. In our patient, RP had been observed much earlier, before the diagnosis of SLE was made. Disorders of the microcirculation in SLE are common and generalized. They affect the skin, cardiovascular system, brain, lungs and glomerular vessels. A systematic review published in 2018 indicates the usefulness of videocapillaroscopy. This simple and non-invasive method is used for the analysis of capillaries in SLE patients. Those authors reported that the capillaroscopic pattern in SLE patients was different from the pattern in healthy individuals and correlated with disease activity. However, a typical capillaroscopic pattern for this disease has not been defined yet [21]. The Reynold's phenomenon (RP) observed in AN is a result of an imbalance between peripheral vasoconstriction and vasodilation due to the increased activity of the sympathetic system in response to stress or emotions. It is suggested that the capillaroscopic pattern in patients with AN and RP is typical of connective tissue diseases [22]. It is possible that the occurrence of RP could be a marker of an increased risk of developing systemic connective tissue diseases in patients with AN. Studies have confirmed that the changes in capillaroscopy always correlate with antiSS-A antibody levels [21]. Our patient had a high level of the antibodies during the observation period. 
Toulany et al. observed that treatment of SLE resulted in remission of AN in all 6 patients who simultaneously developed both diseases [13]. In our patient, several months of glucocorticoid therapy resulted in the resolution of AN symptoms and an improvement in the nutritional status and satisfactory weight gain, which occurred immediately after glucocorticoid therapy and treatment with MMF. In addition, we observed a decrease in immune activity and somatic symptoms of SLE. Improvement in the social functioning of the patient was also noted.

Dysregulation of the immune response which is the basis of chronic inflammation is the indication for permanent glucocorticoid therapy. It may result in adverse effects such as osteoporosis, diabetes, cataracts and cardiovascular complications [23, 24]. Psychiatric side effects (though rarely discussed) include sleep, mood and behavioural disorders, and even the development of psychosis. The severity of glucocorticoid toxicity is dependent on the duration of the therapy and the drug dose. It is also determined by genetic and epigenetic factors and the distribution of glucocorticoid receptors in various tissues [23]. In the case of SLE and AN, glucocorticoid-related adverse effects may mimic or overlap mental disorders that are the symptoms of these diseases. Currently, due to the side effects, researchers focus on searching for alternative treatment options that could be used during exacerbation periods. Flynn et al. presented glucocorticoid-induced leucine zipper (GILZ) which is induced by low concentrations of glucocorticoids and produces anti-inflammatory effects and regulates the function of the immune system. As a result, devastating metabolic adverse effects of glucocorticoid therapy could be avoided [25].

Recently, differences in the composition of the gut microbiome have been observed between patients with $\mathrm{AN}$ and age-matched controls. Morita $e t$ al. reported that patients with AN had significantly higher counts of Clostridium and lower counts of Streptococcus compared to the control group [26]. In addition, Acres et al. hypothesized that abnormal intestinal microflora in patients with AN contributed to the development of cross-reacting autoantibodies that could impair appetite [27]. Impaired food absorption in our patient had already been observed in the first month of our patient's life. Almost one-year hospitalization, antibiotic therapy and introduction of infant formula (modified milk) after birth could have contributed to the disturbance of the intestinal microflora. It is possible that nutrition or hospitalization during infancy could have contributed to the development of both disorders.

In retrospect, the analysis of the disease course suggests that the symptoms of SLE were overlooked. Despite the presence of (at least 2) flares, the patient did not meet all the criteria for SLE and was treated for viral infections. The solution was within reach. However, severe AN was an obstacle to the final diagnosis.

\section{Summary}

Being affected by one severe and chronic disease does not preclude the coexistence of another disease of different aetiology. However, such coexistence may suggest a common pathophysiology. Considering the latest genomic research, adolescents with AN who experienced severe emotional disturbances and neuroticism should be closely monitored for an increased risk of autoimmune diseases. Differential diagnosis should include the coexistence of both disorders and neuropsychiatric SLE. Determination of antineuronal antibodies in the cerebrospinal fluid which are pathognomonic for neuropsychiatric SLE may be helpful in arriving at the final diagnosis.

In our case, immunosuppressive treatment resulted in remission of both diseases, which indicates their common origin. Our observations are consistent with a study by Toulany et al., who observed improvement in all patients with AN as a result of immunosuppressive treatment. Advanced genetic techniques have facilitated the identification of loci on chromosome 12 common to different autoimmune diseases, AN and other neuropsychiatric disorders. This discovery explains the link between these diseases.

The authors declare no conflict of interest.

\section{References}

1. Raevuori A, Haukka J, Vaarala O, et al. (2014): The increased risk for autoimmune diseases in patients with eating disorders. PLoS One 9: e104845.

2. Zerwas S, Larsen JT, Petersen L, et al. (2017): Eating disorders, autoimmune, and autoinflammatory disease. Pediatrics 140: e20162089.

3. Hommer RE, Swedo SE (2017): Anorexia and autoimmunity: challenging the etiologic constructs of disordered eating. Pediatrics 140: e20173060.

4. Słotwińska SM, Słotwiński R (2017): Immune disorders in anorexia. Centr Eur J Immunol 42: 294-300.

5. Treasure J, Zipfel S, Micali N, et al. (2015): Anorexia nervosa. Nat Rev Dis Primers 1: 15074.

6. National Collaborating Centre for Mental Health (UK) (2014): Eating disorders: core interventions in the treatment and management of anorexia nervosa, bulimia nervosa and related eating disorders. British Psychological Society (UK), Leicester (UK) (NICE Clinical Guidelines, No. 9.), Appendix 17. Diagnostic criteria for eating disorders.

7. American Psychiatric Association (2013): Diagnostic and Statistical Manual of Mental Disorders, Fifth Edition: DSM-5.

8. Jagielska G, Kacperska I (2017): Outcome, comorbidity and prognosis in anorexia nervosa. Psychiatr Pol 51: 205-218.

9. Nogal P, Lewiński A (2008): Jadłowstręt psychiczny (anorexia nervosa). Endokr Pol 59: 148-155.

10. Rikani AA, Choudhry Z, Choudhry AM, et al. (2013): A critique of the literature on etiology of eating disorders. Ann Neurosci 20: 157-161. 
11. Hyla-Klekot L, Kucharska G, Słonka K, Karwicka K (2013): Different pathophysiological conditions, clinical picture and therapeutic methods of juvenile systemic lupus erythematosus. Postępy Nauk Medycznych 5: 368-374.

12. Hajdaniuk A, Smoleńska Ż, Nowicka-Sauer K, Zdrojewski Z (2012): Neuropsychiatric syndromes in systemic lupus erythematosus patients. Reumatologia 6: 493-500.

13. Toulany A, Katzman DK, Kaufman M, et al. (2014): Chicken or the egg: anorexia nervosa and systemic lupus erythematosus in children and adolescents. Pediatrics 133: e447-450.

14. Bambery P, Malhotra S, Kaur U, et al. (1987): Anorexia nervosa in a patient with systemic lupus erythematosus. Rheumatol Int 7: 177-179.

15. Sloan D, Gallagher S, Walsh N (1998): Anorexia nervosa complicating systemic lupus erythematosus (SLE). Ir Med J 91: 97.

16. Duncan L, Yilmaz Z, Gaspar H, et al. (2017): Significant locus and metabolic genetic correlations revealed in genome-wide association study of anorexia nervosa. Am J Psychiatry 174: 850-858.

17. Tsokos GC, Lo MS, Costa Reis P, Sullivan KE (2016): New insights into the immunopathogenesis of systemic lupus erythematosus. Nat Rev Rheumatol 12: 716-730.

18. Arbuckle MR, McClain MT, Rubertone MV, et al. (2003): Development of autoantibodies before the clinical onset of systemic lupus erythematosus. N Engl J Med 349: 1526-1533.

19. Fetissov SO, Hallman J, Oreland L, et al. (2002): Autoantibodies against $\alpha-\mathrm{MSH}, \mathrm{ACTH}$ and LHRH in anorexia and bulimia nervosa patients. Proc Natl Acad Sci U S A 99: 17155-17160.

20. Fetissov SO, Harro J, Jaanisk M, et al. (2005): Autoantibodies against neuropeptides are associated with psychological traits in eating disorders. Proc Natl Acad Sci U S A 102: $14865-$ 14870.

21. Cutolo M, Melsens K, Wijnant S, et al. (2018): Nailfold capillaroscopy in systemic lupus erythematosus: A systematic review and critical appraisal. Autoimmun Rev 17: 344-352.

22. De Martinis M, Sirufo MM, Ginaldi L (2018): Raynaud's phenomenon and nailfold capillaroscopic findings in anorexia nervosa. Curr Med Res Opin 34: 547-550.

23. Timmermans S, Souffriau J, Libert C (2019): A General introduction to glucocorticoid biology. Front Immunol 10: 1545.

24. Ciccarelli F, De Martinis M, Ginaldi L (2015): Glucocorticoids in patients with rheumatic diseases: friends or enemies of bone? Curr Med Chem 22: 596-603.

25. Flynn JK, Dankers W, Morand EF (2019): Could GILZ be the answer to glucocorticoid toxicity in lupus? Front Immunol 10: 1684.

26. Morita C, Tsuji H, Hata T, et al. (2015): Gut dysbiosis in patients with anorexia nervosa. PLoS One 10: e0145274.

27. Acres MJ, Heath JJ, Morris JA (2012): Anorexia nervosa, autoimmunity and the hygiene hypothesis. Med Hypotheses 78: 772-775. 